\title{
Polarization switching and nonreciprocity in symmetric and asymmetric magnetophotonic multilayers with nonlinear defect
}

\author{
Vladimir R. Tuz* and Sergey L. Prosvirnin $\dagger$ \\ Institute of Radioastronomy of National Academy of Sciences of Ukraine, \\ 4, Krasnoznamennaya st., Kharkiv 61002, Ukraine and \\ School of Radio Physics, Karazin Kharkiv National University, \\ 4, Svobody Square, Kharkiv 61077, Ukraine \\ Sergei V. Zhukovsky \\ Department of Physics University of Toronto, \\ 60 St. George Street, Toronto, ON, M5S 1A\%, Canada
}

\begin{abstract}
A one-dimensional magnetophotonic crystal with a nonlinear defect placed either symmetrically or asymmetrically inside the structure is considered. Simultaneous effects of time-reversal nonreciprocity and nonlinear spatial asymmetry in the structure are studied. Bistable response is demonstrated in a such system, accompanied by abrupt polarization switching between two circular or elliptical polarizations for transmitted and reflected waves. The effect is explained in terms of field localization at defect-mode spectral resonances and can be used in the design of thin-film optical isolators and polarization transformation devices.
\end{abstract}




\section{INTRODUCTION}

Magnetophotonic crystals (MPC's) are periodic structures that contain magnetic materials and have a period comparable to the wavelength of electromagnetic radiation $\underline{\underline{1}-\underline{6}}$. The simplest example of such a periodic structure is a multilayer having one-dimensional (1D) periodicity. The main advantage of MPC's in contrast to conventional nonmagnetic photonic crystals (PC's) is their possibility to tune the band edge position in the spectrum of the electromagnetic radiation by means of an external static magnetic field. Moreover, the geometric structure of MPC's allows to obtain strong enhancement in a number of magnetooptical effects.

Among the magneto-optical effects that can be significantly enhanced in MPC's, two phenomena are of great interest: (i) the Faraday effect, which denotes rotation of the polarization ellipse of light as it propagates collinearly with an externally applied static magnetic field, and (ii) the nonreciprocity effect, which involves a difference in phase retardation, polarization rotation, and absorption of forward- vs. backward-directed waves propagating through the system.

The Faraday effect can be seen as the lifting of degeneracy for the left (LCP) and right (RCP) circular polarization states, causing the LCP and RCP components to propagate with different phase velocities in the magnetic medium. This difference in velocity of propagation causes the polarization ellipse of the light to rotate as the light propagates. The effect is linear with respect to the static magnetic field strength. The enhancement of this rotation

in MPC's originates from localization of light provided by the multiple interference. $\underline{3}^{\underline{5}} \underline{\underline{5}} \mathrm{In}$ fact, the total rotation angle becomes greater in MPC's with microcavity structure where a magnetic defect is introduced into the periodic system ${ }^{1}$.

Optical nonreciprocity refers to different properties of a medium for electromagnetic waves propagating in opposite directions. It is well known that the nonreciprocity effects are inherent to magnetic media and it can be explained from the symmetry viewpoint $\underline{\underline{\underline{z}}}$ Magnetic field, which is an axial vector, has the symmetry of circular currents set out in a plane perpendicular to its vector. For a medium placed in magnetic field, the rotation directions in this perpendicular plane are non-equivalent. Therefore the optical properties of a magnetic medium are described with a non-symmetric permittivity tensor, and the equations for propagation of LCP and RCP waves in the direction of the field have to be different. 
Thus, circularly polarized waves of opposite handedness (or traveling in opposite directions) are characterized by different phase velocities and/or attenuations in the course of traveling along the same optical path. Since the transformation of a forward-propagating wave into a backward-propagating one with the same handedness is given by the time-reversal operation, the sensitivity of medium properties to the reversal of wave propagation direction is commonly viewed as the time-reversal nonreciprocity.

Aside from the asymmetry of the permittivity tensor, the material nonlinearity can be another source of apparent reciprocity failure. As an example, a nonreciprocal response appears in a layered medium in which frequency changing or self-focusing is asymmetrically located and in which there is also nonuniform dichroism ${ }^{8}$. The order in which the nonlinear and dichroic layers are encountered by incident light will significantly influence the balance between nonlinear and absorptive effects. Another possible way to obtain nonreciprocal response is to combine the nonlinearity in a $\mathrm{PC}$ with an asymmetrically arranged defect (i.e., microcavity) inside it, containing some intensity-dependent material (e.g. a Kerr-type medium). In this system the strong field localization inside the defect can be achieved, and the internal field intensity becomes sufficient to change the optical characteristics of the microcavity through the Kerr effect. Since the spatial field distribution is different for the waves incident on a spatially asymmetric structure from opposite sides, nonreciprocal response appears $\frac{9,10}{}$. It is convenient to call such kind of spatially asymmetric response the reversible nonreciprocity, since no time-reversal symmetry breaking takes place here.

It is also important to note that such nonlinear reversible nonreciprocity is accompanied by optical bistability. Thus, strong field localization in a defect within a PC alters the electromagnetic radiation spectrum including the position of the band edges. This dynamical band edge shift produces optical bistability which consists in the existence of two stable transmission or reflection states for the same input intensity; the typical input-output characteristic of the system contains a hysteresis loop $\underline{11,12}$. In this case the nonreciprocity manifests itself in the different intensity level of input light sufficient to achieve bistable switching for the waves impinging on the system from the opposite sides.

One of the prominent applications of reversible nonreciprocity is the design of a nonlinear electromagnetic diode $\underline{\underline{11}} \underline{\underline{14}}$. On analogy with an electronic diode that transmits electric current in only one direction due to its nonlinear current-voltage characteristics, the nonlinear optical diode features unidirectional transmission of the incoming light. By introducing 
nonlinearity into the MPC, such unidirectional transmission can be achieved for one circular polarization while remaining transparent for the polarization of opposite handedness.

Hence it is of special interest to study the simultaneous effects of time-reversal nonreciprocity and nonlinear spatial asymmetry on the optical properties of PC's. In this paper, we consider an MPC where a nonlinear defect which is placed either symmetrically or asymmetrically inside the periodic structure. An important feature of the studied system is the fact that the asymmetric bistable transmission is accompanied by the polarization conversion $\underline{15} \underline{-17}$. The main objective of our study is focused on achieving the bistabilityinduced abrupt switching between two distinct polarization states. This can be important for thin-film polarization optics devices and polarization-sensitive integrated optics.

The rest of the paper is organized as follows. In Section III, we formulate the problem under study and introduce its solution based on the transfer matrix method of multilayer optics. Sections $\amalg$ and IV follow with the results for a nonlinear defect placed symmetrically and asymmetrically into an MPC, respectively. Finally, Section $\nabla$ summarizes the paper.

\section{PROBLEM FORMULATION AND SOLUTION}

We consider a planar multilayer stack of infinite transverse extent (Fig. 11). Each unit cell is composed of a bilayer which consists of magnetic (with constitutive parameters $\varepsilon_{1}, \hat{\mu}_{1}$ ) and nonmagnetic (with parameters $\varepsilon_{2}, \mu_{2}$ ) layers. The magnetic layers are magnetized up to saturation by an external static magnetic field $\vec{M}$ directed along the $z$-axis (Faraday configuration). A defect is created by introducing into the structure a layer with constitutive parameters $\varepsilon_{d}, \mu_{d}$. We assume that this layer is a Kerr nonlinear dielectric, which permittivity $\varepsilon_{d}$ linearly depends on the intensity $|E|^{2}$ of the electric field. The defect can be settled either symmetrically or asymmetrically in the middle of the structure. The parameters $m$ and $n$ describe the number of bilayers placed before and after the defect layer. In any case the bilayers are arranged symmetrically with respect to the defect layer, i.e. the structure begins and ends with layers of the same type. We suppose that all layers have the same thickness $D$. The outer half-spaces $z \leq 0$ and $z \geq[2(m+n)+1] D$ are homogeneous, isotropic, and have parameters $\varepsilon_{0}, \mu_{0}$. Assume that the normally incident field is a linearly polarized plane monochromatic wave of a frequency $\omega$ and an amplitude $A$. For the sake of

definiteness, we also suppose that the vector $\vec{E}$ of the incident wave is directed along the 
$x$-axis.

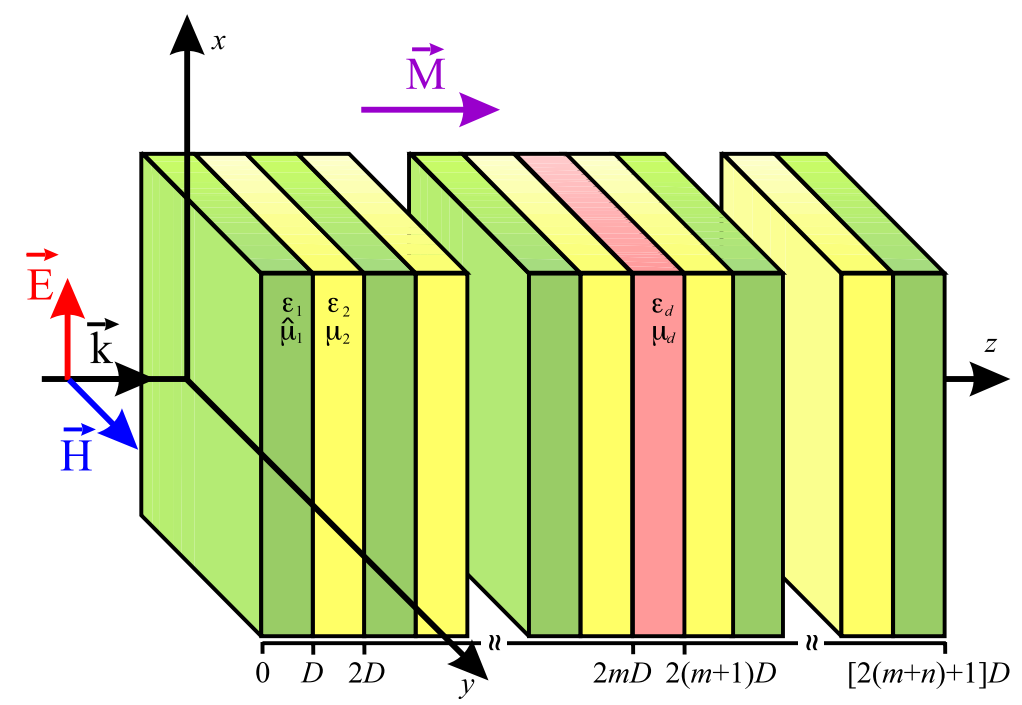

FIG. 1: (Color online) Magnetophotonic structure with nonlinear defect.

As an convenient material for magnetic layers, the family of impurity-doped yttriumiron garnet ( $\mathrm{YIG}$ ) $\mathrm{Y}_{3} \mathrm{Fe}_{5} \mathrm{O}_{12}$ films can be proposed. These magnetic oxides are well studied and widely used in integrated magneto-optics because they are transparent in the near infrared region $\underline{3, \underline{5}}$. As an example, a few types of multilayered films composed of magnetic Bisubstituted YIG (Bi:YIG) and dielectric $\mathrm{SiO}_{2}$ or glass FR-5 layers were investigated. 2.7 The MPC's based on the other materials are also known. Thus, a new class of semiconductormagnetic hybrid nanostructures consist of GaAs with MnAs nanoclasters (GaAs:MnAs) which are paired with GaAs/AlAs superlatices is recently investigated experimentally in the range 900-1100 $\mathrm{nm} \frac{18}{}$. Also in the nonlinear regime the structure based on the semimagnetic semiconductors such as $\mathrm{Cd}_{1-x} \mathrm{Mn}_{x}$ Te with the defect being a quantum well with prescribed spectral characteristics was reported $\underline{19}, 20$. From these papers it may be deduced that the magnetic materials manifest their nonlinear properties at the light intensity about $1 \mathrm{GW} / \mathrm{cm}^{2}$. In our present paper we consider the nonlinear defect which is made of nonmagnetic material due to its greater availability. As an example, AsGa or InSb can be selected for this purpose. We prefer such structure configuration because these materials require much lower intensities of the incident light to enable the nonlinear effects. From the literature ${ }^{21}$ it can be deduced that the nonlinear response in the semiconductor materials can be achieved at the light intensity about $1 \mathrm{~kW} / \mathrm{cm}^{2}$. Although a defect is made of nonmagnetic material, the studied structure that consists of magnetic layers and such nonlinear defect exhibits a 
number of very interesting and unique properties that we consider.

Our solution is based on the transfer matrix formalism ${ }^{22}$ which is used to calculate the field distribution inside the structure and the reflection and transmission coefficients of the MPC. In the Faraday configuration, when external static magnetic field is biased parallel to the direction of wave propagation $(\vec{k} \| \vec{M})$, the magnetic permeability $\hat{\mu}_{1}$ is a tensor quantity with nonzero off-diagonal components:

$$
\hat{\mu}_{1}=\left(\begin{array}{ccc}
\mu_{1}^{T} & i \alpha & 0 \\
-i \alpha & \mu_{1}^{T} & 0 \\
0 & 0 & \mu_{1}^{L}
\end{array}\right)
$$

For the description of electromagnetic waves in this case it is necessary to use a $4 \times 4$ transfer matrix formulation 23 . Thus, at the first stage, in the linear case, the equation which defines the coupling of the tangential field components at the input and output of the structure is written in the next form 24,25

$$
\vec{\Psi}(0)=\mathfrak{M} \vec{\Psi}(\Lambda)=\left\{\left(\mathbf{M}_{1} \mathbf{M}_{2}\right)^{m} \mathbf{M}_{d}\left(\mathbf{M}_{2} \mathbf{M}_{1}\right)^{n}\right\} \vec{\Psi}(\Lambda)
$$

where $\vec{\Psi}=\left\{E_{x}, E_{y}, H_{x}, H_{y}\right\}^{T}$ is the vector containing the tangential field components at the structure input and output; the upper index $T$ is the matrix transpose operator; $\Lambda$ is the total length of the structure, $\Lambda=[2(m+n)+1] D ; m$ and $n$ are the numbers of periods placed before and after the defect element; $\mathbf{M}_{1}, \mathbf{M}_{2}$ and $\mathbf{M}_{d}$ are the transfer matrices of the rank four of the first, second, and defect layers, respectively. The elements of the transfer matrices in (11) are determined from the solution of the Cauchy problem and are given in ${ }^{23}$.

As the solution of the linear problem (11) is obtained, the intensity of the reflected and transmitted fields and the distribution of the field $\vec{E}_{i n}(z)$ inside the MPC can be calculated. Generally, when the defect layer consists of a Kerr nonlinear dielectric, the permittivity $\varepsilon_{d}$ is inhomogeneous, and depends on the intensity of the electric field at each point of this layer as follows

$$
\varepsilon_{d}(z)=\varepsilon_{d}^{l}+\varepsilon_{d}^{n l}\left|E_{i n}(z)\right|^{2},(2 m D \leq z \leq(2 m+1) D) .
$$

Knowing the field intensity in the defect layer, both the actual value of permittivity $\varepsilon_{d}$ and, consequently, the actual value of transfer-matrix $\mathfrak{M}$ can be calculated. Thus we deal with an equation on the unknown function of field intensity distribution inside the defect layer. A magnitude of the incident field $A$ is an independent parameter of this equation. Since 
the parameter $\varepsilon_{d}^{n l}$ is small and the nonlinear contribution to $\varepsilon_{d}$ varies with the longitudinal distance on the scale of one-half wavelength we provide an approach which regards $\varepsilon_{d}$ as independent on $z$ and treats the dependence of $\varepsilon_{d}$ on the average intensity of the electric field $\overline{\left|E_{i n}\right|^{2}}$ inside the defect layer. Quantitative reasoning of this approach is presented in ${ }^{25}$. On the basis of this approximation, we suppose that the permittivity of the medium depends on the average intensity of the electric field as $\varepsilon_{d}=\varepsilon_{d}^{l}+\varepsilon_{d}^{n l} \mid \overline{\left.E_{i n}\right|^{2}}$.

As a result, at the second stage, the nonlinear equation related to the average field intensity distribution in the defect is obtained. The numerical solution of this equation yields us the final field distribution in the MPC and the values of the reflection $R$ and transmission $T$ coefficients, which expressions can be found in $\underline{23}$.

\section{SYMMETRIC MULTILAYERS: POLARIZATION BISTABILITY}

Our objective here is to study the main features of optical response for an MPC with a nonlinear defect placed symmetrically inside it. For this reason we consider an MPC consisting of two sections with the same number of bilayers in them $(m=n)$. The sections are located symmetrically on each side of the defect layer. The main idea of such an arrangement is to obtain a significant field localization inside the defect layer, which is achieved by an appropriate choice of the number of periods $m$ and the material parameters of layers.

The basic optical properties of the studied MPC are inherited from the characteristics of perfectly periodic structures with nonmagnetic layers. Recall that all periodic structures with layer thicknesses comparable to the wavelength possess forbidden frequency gaps (stopbands or band gaps) as a direct consequence of Floquet-Bloch theorem $\underline{26}^{2}$ These gaps are determined by the modulation period and the average refractive index. Propagation of waves with frequencies in the stopbands of an idealized infinite structure is completely inhibited, and the band gaps are in this sense perfect. For finite structures these gaps appear as frequency regions with low transmittance and high reflectance, located between high-transmittance passbands. If any distortion (a "defect") is introduced inside a periodic structure, transmission resonances can appear in the stopbands, with the field strongly localized in the defect. The existence of such "localization resonances" is explained by the fact that the defect forms a resonant Fabry-Perot cavity enclosed between two Bragg mirrors.

The main distinctive feature of an MPC in contrast to the nonmagnetic one is the ap- 

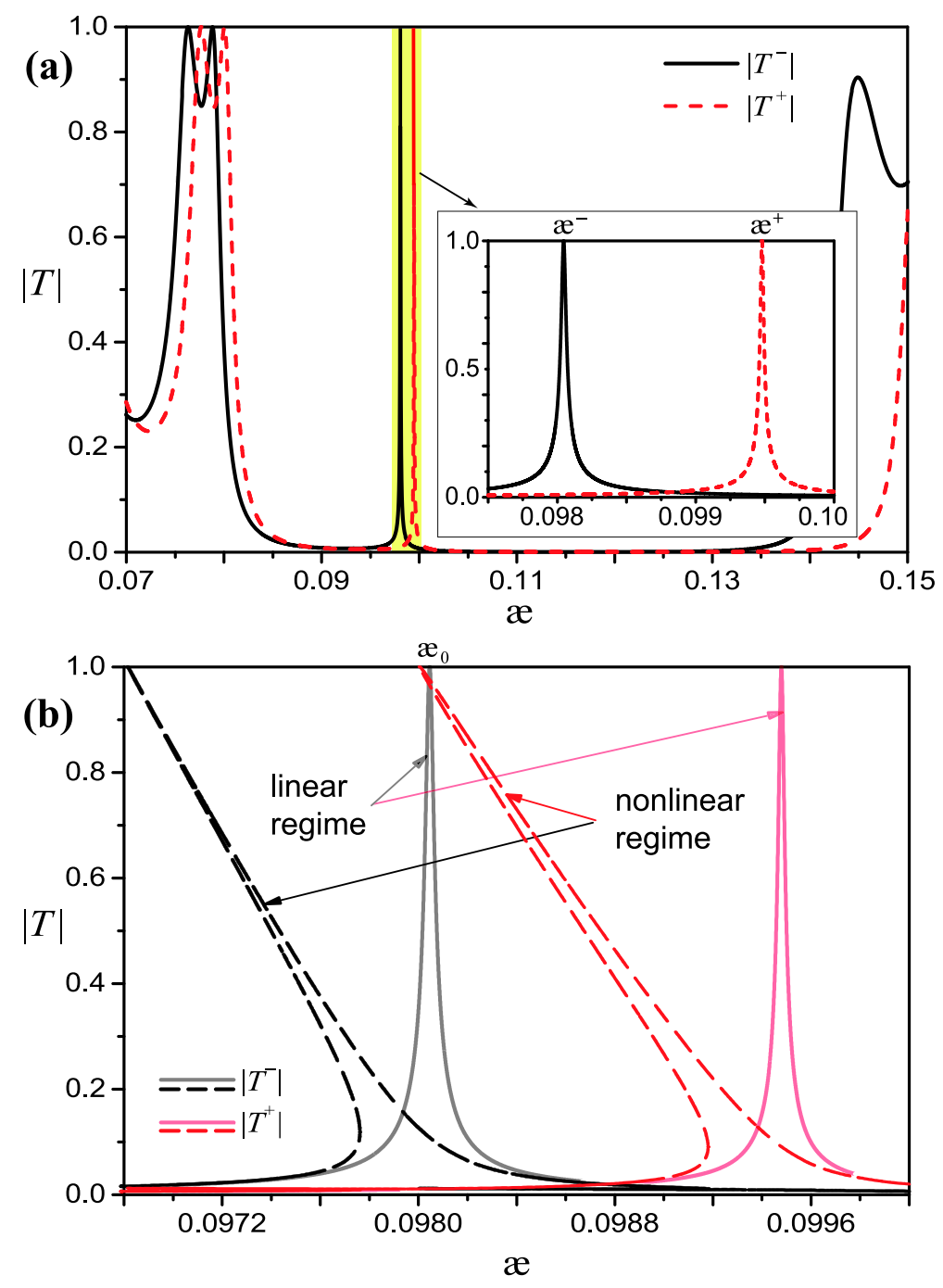

FIG. 2: (Color online) Frequency dependences $(\varkappa=D / \lambda)$ of the transmission coefficient $(T)$ of the LCP $(-)$ and RCP $(+)$ waves in the (a) linear (b) nonlinear case for $m=n=5, \varepsilon_{1}=10$, $\mu_{1}^{T}=\mu_{1}^{L}=1, \alpha=0.05, \varepsilon_{2}=\mu_{2}=\mu_{d}=1, \varepsilon_{d}^{l}=4$. For the nonlinear case, $\tilde{\varepsilon}_{d}^{n l}=\varepsilon_{d}^{n l} I_{0}=1.5 \times 10^{-4}$, which corresponds to the incident light intensity $I_{0}=15 \mathrm{~kW} / \mathrm{cm}^{2}$ for $\varepsilon_{d}^{n l} \simeq 1.0 \times 10^{-5} \mathrm{~cm}^{2} / \mathrm{kW}$.

pearance of circular polarization eigenstates. Such circular polarization eigenstates are also inherent in PC's with chiral isotropic layers 24,25 but in the case of MPC's they are controlled with an external static magnetic field. Thus the MPC reacts differently to circularly polarized waves with opposite handedness, with distinct optical spectra for each of them (see Figs. $2 \mathrm{a}$ and $3 \mathrm{a}$ ). This way, in the Faraday configuration, both the edges of the forbidden bands and the frequencies of the localized defect modes become different for LCP vs. RCP incident wave. As a result, the defect resonances split into doublets (see Figs. 2a -3) known as 



FIG. 3: (Color online) Same as Fig. 2 for the reflection coefficient $(R)$.

the longitudinal Zeeman-like doublets $\frac{16}{}$. These doublets originate from lifting of the degeneracy between resonant conditions for the LCP and RCP waves in the underlying MPC by the external magnetic field. It can be seen that there are two closely spaced resonant modes in the stopband, one of which is an RCP eigenmode and the other is an LCP eigenmode.

In the insets of Figs. 23 the frequency band where the doublet exists is given on a larger scale. Throughout the paper we suppose that the working frequency is far from the frequency of the ferromagnetic resonance of magnetic layers and their losses are negligibly small. Under this assumption, at the resonant frequencies, the magnitude of the transmission coefficient of the corresponding circularly polarized mode reaches unity, and the structure becomes completely transparent for the LCP wave when $\varkappa^{-} \approx 0.098$ and for the RCP wave 
when $\varkappa^{+} \approx 0.0995$. Obviously, the magnitude of splitting (the frequency difference between the peaks $\left.\Delta \varkappa=\varkappa^{+}-\varkappa^{-}\right)$can be easily tuned by changing the strength of the external static magnetic field.

Now we consider the case when the MPC contains a Kerr-type nonlinear defect. It is known that the introduction of such a defect into an otherwise linear structure can induce bistable behavior in the system. The nature of this bistability is studied in the theory of the nonlinear Fabry-Perot resonators quite well. 27 The resonant frequencies $\varkappa^{ \pm}$are sensitive to the refractive index of the material within the cavity. Thus, when the frequency of the incident wave is tuned near a resonant frequency, the field localization induces growth of the light intensity inside the cavity, which, by means of the Kerr effect, eventually alters the refractive index enough to shift the resonant frequency. When this shift brings the resonant condition closer to match the frequency of the incident field, even more energy gets localized in the cavity. This further enhances the shift of the resonance, creating positive feedback that leads to formation of a hysteresis loop in the spectra with respect to the incident field intensity. As a result, for a fixed input field intensity, the frequency dependences for any resonant mode have a typical shape of "bent resonances". In the spectra of a nonlinear MPC this bending can be seen for both resonant modes in the split doublets (Figs. 2 $\mathrm{b}-3 \mathrm{~b}$ ).

Now consider a linearly polarized wave incident on an MPC with defect. One can represent it as a superposition LCP and RCP waves. As a result, the corresponding optical spectra will contain both resonances. This is demonstrated in Fig. 4 for individual polarization components of reflected and transmitted light, as measured in typical experiments. Since the whole system possesses axial symmetry in the considered case of normal incidence and Faraday configuration, we can only distinguish between co-polarized (e.g., ss or $p p$, denoted $c o$ ) and cross-polarized ( $s p$ or $p s$, denoted $c r$ ) components. Since LCP and RCP cmponents are present in a linearly polarized wave in equal proportion, the magnitudes of the co-polarized and cross-polarized components are equal to each other at the resonant frequencies, $\left|T^{c o}\right|=\left|T^{c r}\right|=\left|R^{c o}\right|=\left|R^{c r}\right|=0.5$. These conditions are satisfied in the both linear and nonlinear regimes. In the nonlinear case, both localization resonances are bent. The "angle" of bending clearly depends on the intensity of the incident field and is almost the same for both resonances in the doublet.

Due to the above mentioned polarization sensitivity of a magnetophotonic system, a linearly polarized wave will very likely undergo a change in its polarization state during 

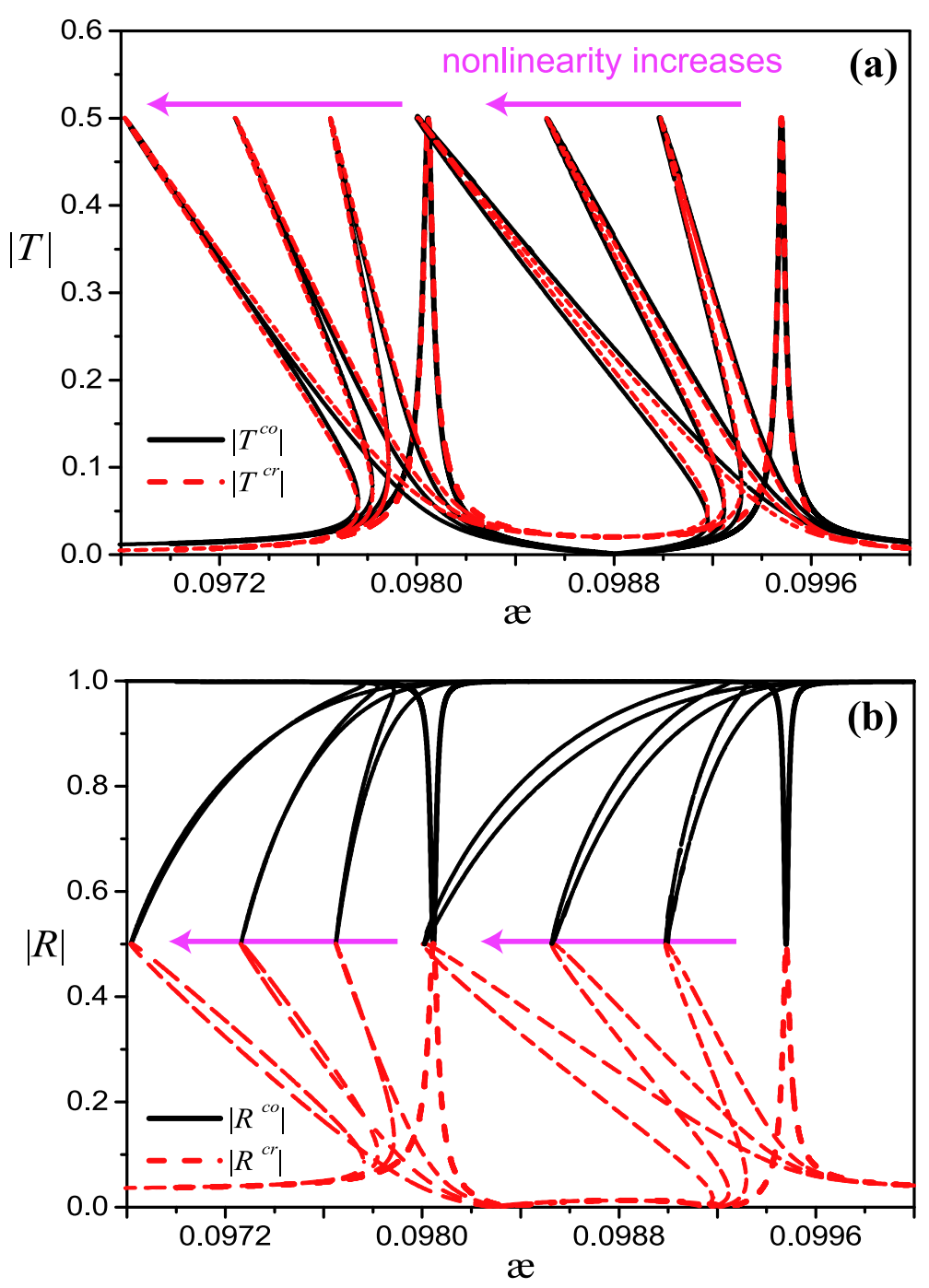

FIG. 4: (Color online) Frequency dependences $(\varkappa=D / \lambda)$ of the magnitudes of the co-polarized (co) and cross-polarized (cr) components of the transmission (a) and reflection (b) coefficients of linearly polarized waves. The input intensity $I_{0}$ in the nonlinear regime is taken to be 5,10 , and $15 \mathrm{~kW} / \mathrm{cm}^{2}$. Other parameters are as in Fig. 2.

reflection or transmission. This is confirmed in Fig. 5, which shows the corresponding frequency dependences of the ellipticity angle $(\eta)$ and the polarization azimuth $(\theta)$ for the transmitted (black lines) and reflected (red lines) fields. According to the definition of the Stokes parameters, we introduce the ellipticity $\eta$ so that the field is linearly polarized when $\eta=0$, and $\eta=-\pi / 4$ for LCP and $+\pi / 4$ for RCP (note that in the latter cases the preferential azimuthal angle of the polarization ellipse $\theta$ becomes undefined). In all other cases $(0<|\eta|<\pi / 4)$, the field is elliptically polarized. In the considered frequency band and 



FIG. 5: (Color online) Frequency dependences $(\varkappa=D / \lambda)$ of (a) the elipticity angle $\eta$ and (b) the polarization azimuth $\theta$ of the transmitted and reflected fields. The incident light is linearly polarized, and structure parameters are as in Fig. 2, The vertical line marks the bistable polarization switching at $\varkappa_{0}$.

in the linear regime, the transmitted field experiences the rotation of its polarization ellipse and sequentially changes between LCP and RCP through elliptical and linear polarization states (Fig. [4, solid black lines). On the contrary, the reflected field is linearly polarized almost in the whole selected band except the frequencies $\varkappa^{-}$and $\varkappa^{+}$where it becomes circularly polarized (Fig. 5, solid red lines). Note that at these resonant frequencies the polarization azimuth $\theta_{r e f}=\theta_{r e f}(\varkappa)$ is a discontinuous function. 
Such a drastic difference in the polarization states of the transmitted vs. reflected fields can be understood from the fact that the operating frequencies lie in the stopband of the MPC where an impinging wave is almost completely reflected from the structure. As the incident field is linearly polarized, so, too, is the reflected field. Due to the finite size of the structure a small fraction of the wave's energy still gets transmitted through the MPC, undergoing a $90^{\circ}$ rotation of its polarization ellipse (Fig. 5b) for $\varkappa^{-}<\varkappa<\varkappa^{+}$. At the resonant frequencies, it is evident that the matching circularly polarized eigenmode passes through the system while for the orthogonally polarized eigenmode the transmission is still forbidden. Therefore, both transmitted and reflected fields become circularly polarized within the localized modes frequencies. Note that the reflected field has the same polarization state as the transmitted field because the reflected wave propagates in the opposite direction (see Ref. 7 for clarity).

In the nonlinear regime the ellipticity angle and the polarization azimuth become multivalued functions. Therefore, it is possible to use multistability to switch not only between different transmittances and reflectances but also between two (or, generally, more than two) distinct polarization states in the transmitted and/or reflected light.

The most intriguing scenario for such switching is expected when a bent resonance at $\varkappa^{+}$spectrally overlaps with the original location of the other resonance at $\varkappa^{-}$. This overlap is possible as the resonances are spectrally close to each other. For example, let us fix the operating frequency $\varkappa_{0}$ at $\varkappa^{-}$. At this frequency the reflected and transmitted fields ought to be LCP. As the intensity of input field rises, the other resonance corresponding to $\varkappa^{+}$ and associated with RCP undergoes red shift and eventually reaches $\varkappa_{0}$. It becomes possible to couple the incident wave with frequency $\varkappa_{0}$ with either of the eigenmodes. Since these have opposite circular polarizations (they are associated with converting a linearly polarized incident light into LCP and RCP), it can be expected that switching between these two polarization states can be achieved.

Indeed, Fig. 5 shows that at a frequency $\varkappa_{0} \approx \varkappa^{-}$the bistable switching occurs between $\mathrm{RCP}$ and near-LCP for the transmitted light and between linear polarization and RCP for the reflected light. This agrees with the above explanation and is seen in the behaviour of resonance bending in the Stokes parameter space (Fig. 5). For the reflected light the bending in ellipticity resembles that in the reflectance (Fig. 4a). For the transmitted light the bent resonances occur in the immediate vicinity of $\eta= \pm \pi / 4$, because only circularly 
polarized waves can fully couple to the MPC eigenmodes to become transmitted through it.

Finally, note that Fig. 4b illustrates another peculiarity of the reflection spectra of the structure under study, namely, the formation of closed loops, which appear in the crosspolarized component of the reflected field. In particular, the closed loop appears in the lower-frequency resonance at $\varkappa^{-}$. The physical mechanism of loop formation is the difference between the values of $T_{c o}$ and $R_{c o}$ to either side of the resonance. In the linear regime, $\left|T_{c o}\left(\varkappa^{-}-\delta\right)\right|<\left|T_{c o}\left(\varkappa^{-}+\delta\right)\right|$ since transmittance between the resonances should be higher that to the either side of both defects because it is influenced by the Lorentzian tails of both resonances. Consequently,

$$
\left|R_{c o}\left(\varkappa^{-}-\delta\right)\right|>\left|R_{c o}\left(\varkappa^{-}+\delta\right)\right|
$$

(This inequality can also be influenced by non-symmetric placement of the resonances in the band gap due to the violation of the quarter-wave condition in the structures under study.) In the nonlinear regime, the relation in Eq. (3) holds, and the resonance bending to the direction of lower frequencies will cause a loop to form.

\section{ASYMMETRIC CONFIGURATION: POLARIZATION CONVERSION}

Nonlinear multilayer structures with spatial asymmetry, are commonly considered to obtain directional sensitivity or reversible nonreciprocity in nonmagnetic PC's. As a few examples, random or deterministically aperiodic media, as well as periodic structures with asymmetrically positioned defects, were recently reported to have direction-dependent or unidirectional transmission. $11,12,14,25,28,29$ The general result is that interaction between nonlinearity and asymmetry manifests itself in the simultaneous occurrence of bistability (or multistability) and nonreciprocity.

From a mathematical point of view, this all-optical reversible nonreciprocity is a result of non-commutativity of matrix multiplication in Eq. (1) when the transfer-matrix of the structure is calculated. In partuicular, optical properties of a 1D periodic structure with a defect strongly depend on the position of that defect layer inside the sample. Nevertheless, in the linear regime, specific properties of the transfer matrix that stem from time-reversal reciprocity of the Maxwell equations ensure that the transmission through the system re-

mains the same regardless of whether the field is incident from the left or right side of the 




FIG. 6: (Color online) Frequency dependences $(\varkappa=D / \lambda)$ of the transmission $(T)$ and reflection $(R)$ coefficients of the LCP $(-)$ and $\mathrm{RCP}(+)$ waves of the MPC with asymmetrically placed $(m \neq n)$ nonlinear defect. Here, $\tilde{\varepsilon}_{d}^{n l}=\varepsilon_{d}^{n l} I_{0}=1.0 \times 10^{-4}$, i.e., $I_{0}=10 \mathrm{~kW} / \mathrm{cm}^{2}$ for $\varepsilon_{d}^{n l} \simeq 1.0 \times 10^{-5} \mathrm{~cm}^{2} / \mathrm{kW}$. Other parameters are as in Fig. 2. Solid and dash lines correspond to $(m=5, n=6)$ and $(m=6$, $n=5)$ configurations, respectively.

structure.

The situation changes drastically if an optically sensitive (e.g., Kerr-type nonlinear) material is used for the defect layer. In this case, due to different field localization patterns within the defect layer for the waves impinging from the left and right sides of the structure, the nonlinear response becomes different. This difference manifests itself in the different angles of bending of the localization resonances. 12

Our goal here is to study the simultaneous effect of the spatial asymmetry and the time-reversal nonreciprocity on the behavior of the localization resonances in the MPC. We modify the structure from Section [II to make the number of bilayers in two subsections before and after the defect element different $(m \neq n)$. We additionally assume that the static magnetic field direction always coincides with the wave propagation direction. This can be assumed without loss of generality because changing the direction of wave propagation without changing the direction of the static magnetic field reverses the handedness of the circularly polarized states $(\mathrm{RCP} \rightleftharpoons \mathrm{LCP})$. Hence by considering the response of the original structure characterized by $(m, n)$ and its mirror-symmetric counterpart $(n, m)$ to LCP and 

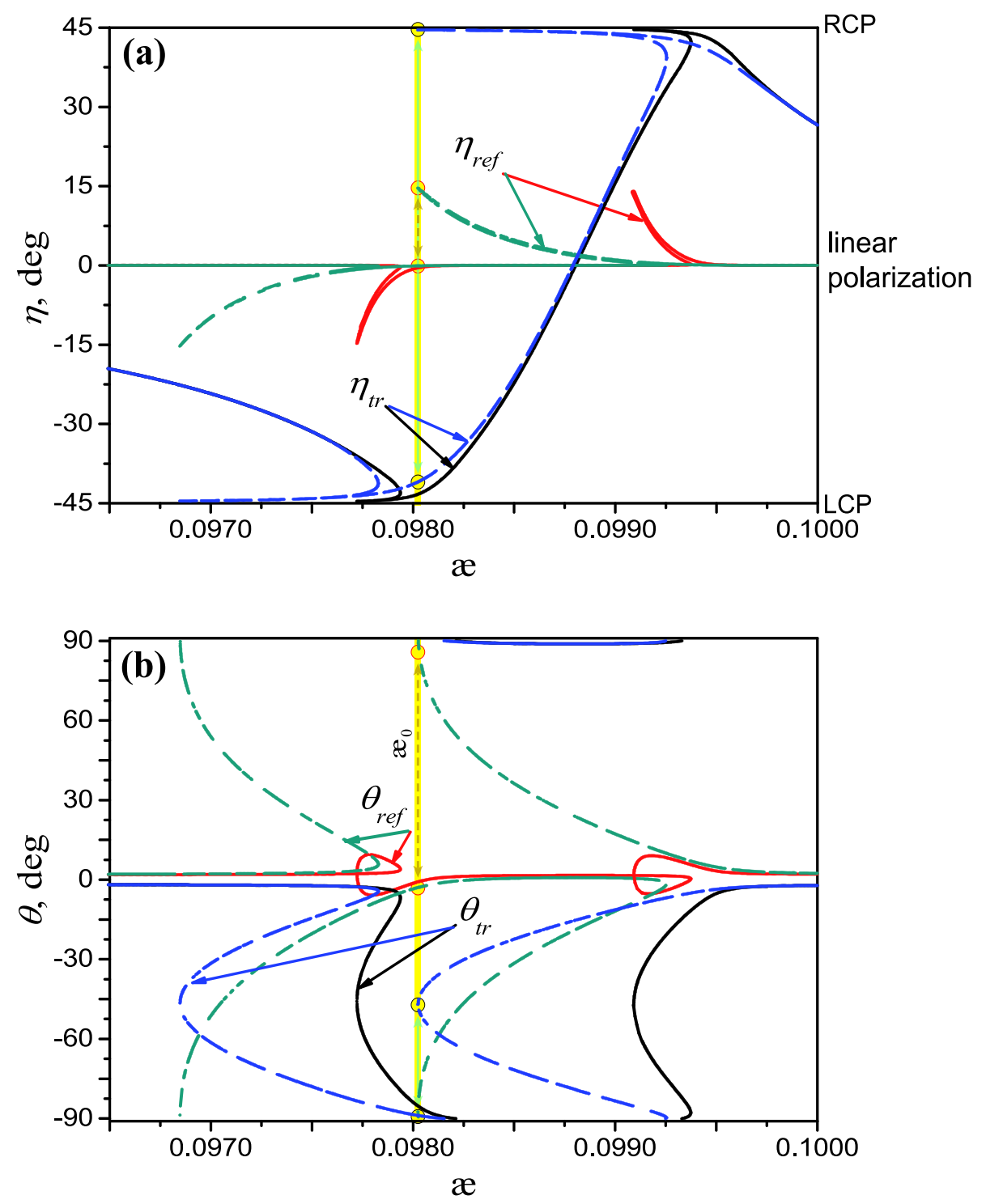

FIG. 7: (Color online) Frequency dependences $(\varkappa=D / \lambda)$ of the elipticity angle (a) and the polarization azimuth (b) of the transmitted and reflected fields of the MPC with asymmetrically placed $(m \neq n)$ nonlinear defect. Parameters are as in Fig. 6. The vertical line marks the bistable polarization switching at $\varkappa_{0}$.

RCP incident wave solves the problem completely.

Comparison of the results presented in Figs. $2 \mathrm{~b}-3 \mathrm{~b}$ and Fig. 6 shows that adding one bilayer at either side of the MPC drastically changes the spectra of the structure. These changes are associated with the already mentioned different field distribution inside the structure. The stark difference in the angles of the localization resonance bending results from the all-optical reversible nonreciprocity. 



FIG. 8: (Color online) Frequency dependences $(\varkappa=D / \lambda)$ of the magnitudes of the co-polarized (co) and cross-polarized (cr) components of the transmission (a) and reflection (b) coefficients of the linearly polarized waves of the MPC with asymmetrically placed $(m \neq n)$ nonlinear defect. Parameters are as in Fig. 6.

The accompanying change of the magnitude for the reflection and transmission coefficients at the bent resonances (so that $\left|T_{\max }^{ \pm}\right|<1$ and $\left|R_{\min }^{ \pm}\right|>0$ ) results from a certain conflict in the design principles for resonant multilayers. Namely, to increase the structure's sensitivity to the direction of incidence, one needs to increase its the spatial asymmetry; yet to increase the maximum transmission at a resonant peak, the structure should remain close to symmetric $22 \underline{28}$. As a consequence, at the frequencies of the localization resonances the transmission is always below unity and the reflected field is always elliptically rather than circularly polarized. Indeed, as seen in Fig. 7, the ellipticity angle $\left|\eta_{\text {ref }}\right|<\pi / 4$ in the whole 
selected frequency band. The transmitted field is still circularly polarized at the localization resonances. The polarization azimuth $\theta_{\text {ref }}=\theta_{\text {ref }}(\varkappa)$ is now a continuous function. Hence, while the symmetric structure features polarization switching between two circularly polarized states, the asymmetric one only enables switching between two elliptically polarized states.

However, it can be seen that changing the position of the defect layer within the structure significantly alters the ratio between the reflected and transmitted field, and in particular the relations between co-polarized and cross-polarized components in them (Fig. 8). While the magnitudes of the co-polarized and cross-polarized transmission components remain equal to each other $\left(\left|T^{c o}\right|=\left|T^{c r}\right| \leq 0.5\right)$, the the relation between the reflection components $\left(\left|R^{c o}\right|\right.$ and $\left.\left|R^{c r}\right|\right)$ varies in a much wider range. In one structure configuration $(m=5$, $n=6$ ), the peak magnitudes of the co-polarized and cross-polarized reflection components are $\left|R^{c o}\right| \approx 0.8$ and $\left|R^{c r}\right| \approx 0.2$. In the other configuration $(m=6, n=5)$ they are opposite: $\left|R^{c o}\right| \approx 0.2$ and $\left|R^{c r}\right| \approx 0.8$. In the latter case there is an obvious significant polarization transformation in the reflected field so that a $90^{\circ}$ polarization rotation of the incident light can be achieved with good conversion efficiency. This can find useful application as thinfilm tunable polarization-rotating mirrors. Also, an appropriate choice of the asymmetric structure configuration, material parameters, layer thicknesses, and magnetic field strength would achieve switching between two orthogonal linear polarization states in the reflected field. This can be important in the design of tunable thin-film polarization splitters and switchers.

\section{CONCLUSIONS}

In the present paper, we have studied the effects of bistability, nonreciprocity, and polarization transformation in a magnetophotonic crystal with a nonlinear defect placed either symmetrically or asymmetrically inside the structure. The problem is considered in the Faraday configuration, i.e, the external static magnetic field is applied in the direction of the structure periodicity and is collinear with the wave vector of the incident wave.

The reflection and transmission coefficients of the structures, along with the field distribution inside them, are calculated using the transfer matrix approach. The nonlinear problem is solved under the assumption that the nonlinear permittivity of the medium inside the 
defect layer depends on the average intensity of the electric field inside the defect.

In the case of symmetric structure configuration, it is shown that a bistable response of a nonlinear magnetophotonic system features switching between two circular polarization states within the localization resonances (defect modes) for reflected and transmitted fields. In the case of asymmetric structure configuration, this switching appears between elliptically polarized states in the reflected field, and between circularly polarized states in the transmitted field. The asymmetric structure also features strong $90^{\circ}$ polarization rotation in the reflected field, with a potential for bistable switching between linear polarizations.

From the specific parameters used in our numerical calculations, it is reasonable to conclude that bistable response and stepwise polarization switching can already be achieved at the incident power densities of $10-100 \mathrm{~kW} / \mathrm{cm}^{2}$ with available materials in the considered structure configuration.

* tvr@rian.kharkov.ua

$\dagger$ prosvirn@rian.kharkov.ua; http://ri.kharkov.ua/prosvirn/

$\ddagger$ szhukov@physics.utoronto.ca

1 M. Inoue, K. Arai, T. Fujii, and M. Abe, J. Appl. Phys. 85, 5786 (1999).

2 S. Sakaguchi and N. Sugimoto, J. Opt. Soc. Am. A 16, 2045 (1999).

3 I. L. Lyubchanskii, N. N. Dadoenkova, M. I. Lyubchanskii, E. A. Shapovalov, and T. Rasing, J. Phys. D: Appl. Phys. 36, R277 (2003).

4 V. I. Belotelov and A. K. Zvezdin, J. Opt. Soc. Am. B 22, 286 (2005).

5 M. Inoue, R. Fujikawa, A. Baryshev, A. Khanikaev, P. B. Lim, H. Uchida, O. Aktsipetrov, A. Fedyanin, T. Murzina, and A. Granovsky, J. Phys. D: Appl. Phys. 39, R151 (2006).

6 S. V. Chernovtsev, D. P. Belozorov, and S. I. Tarapov, J. Phys. D: Appl. Phys. 40, 295 (2007).

7 A. K. Zvezdin and V. A. Kotov, Modern Magnetooptics and Magnetooptical Materials (Institute of Physics Publishing, Bristol and Philadelphia, 1997).

8 R. J. Potton, Rep. Prog. Phys. 67, 717 (2004).

9 E. Lidorikis, K. Busch, Q. M. Li, C. T. Chan, and C. M. Soukoulis, Phys. Rev. B 56, 15090 (1997).

10 L. Diao and S. Blair, J. Opt. A: Pure Appl. Opt. 9, 972 (2007). 
11 V. Grigoriev and F. Biancalana, New J. Phys. 12, 053041 (2010).

12 S. V. Zhukovsky and A. G. Smirnov, Phys. Rev. A 83, 023818 (2011).

13 P. Hou, Y. Chena, J. Shia, M. Shena, and Q. Wang, Opt. Commun. 273, 441 (2007).

14 A. E. Miroshnichenko, E. Brasselet, and Y. S. Kivshar, Appl. Phys. Lett. 96, 063302 (2010).

15 F. Jonson and C. Flytzanis, J. Nonlinear Opt. Phys. Mat. 13, 129 (2004).

16 F. Jonson and C. Flytzanis, Phys. Rev. Lett. 96, 063902 (2006).

17 I. L. Lyubchanskii, N. N. Dadoenkova, A. E. Zabolotin, Y. P. Lee, and T. Rasing, J. Appl. Phys. 103, 07B321 (2008).

18 H. Shimizu, M. Miyamura, and M. Tanaka, Appl. Phys. Lett. 78, 1523 (2001).

19 C. Buss, R. Pankoke, P. Leisching, J. Cibert, R. Frey, and C. Flytzanis, Phys. Rev. Lett. 78, 4123 (1997).

20 D. P. Cubian, M. Haddad, R. André, R. Frey, G. Roosen, J. L. A. Diego, and C. Flytzanis, Phys. Rev. B 67, 045308 (2003).

21 E. D. Palik, Handbook of Optical Constants of Solids (Academic Press, Boston, 1991).

22 D. W. Berreman, J. Opt. Soc. Am. 62, 502 (1972).

23 V. Tuz, M. Vidil, and S. Prosvirnin, J. Opt. 12, 095102 (2010).

24 V. Tuz, J. Opt. Soc. Am. B 26, 1693 (2009).

25 V. Tuz and S. Prosvirnin, J. Opt. Soc. Am. B 28, 1002 (2011).

26 K. Sakoda, Optical Properties of Photonic Crystals (Springer-Verlag, Berlin, 2005).

27 H. M. Gibbs, Optical Bistability: Controlling Light with Light (Academic Press, Orlando, Fla., $1985)$.

28 S. V. Zhukovsky, Phys. Rev. A 81, 053808 (2010).

29 I. V. Shadrivov, K. Y. Bliokh, Y. P. Bliokh, V. Freilikher, and Y. S. Kivshar, Phys. Rev. Lett. 104, $123902(2010)$. 